\title{
Visual Distraction: An Altered Aiming Spatial Response in Dementia
}

\author{
Elizabeth E. Galletta Anthony H. Lequerica Scott R. Pekrul \\ Paul J. Eslinger Anna M. Barrett
}

Kessler Foundation Research Center, West Orange, N.J., USA

\section{Key Words}

Visual distraction - Line bisection - Where error - Aiming error

\begin{abstract}
Background/Aims: Healthy individuals demonstrate leftward bias on visuospatial tasks such as line bisection, which has been attributed to right brain dominance. We investigated whether this asymmetry occurred in patients with probable dementia of the Alzheimer type (pAD) which is associated with neurodegenerative changes affecting temporoparietal regions. Methods: Subjects with PAD and matched controls performed a line bisection task in near and far space under conditions of no distraction, left-sided visual distraction and right-sided visual distraction. Results: Participants with PAD manifested different motor-preparatory 'aiming' spatial bias than matched controls. There were significantly greater rightward 'aiming' motor-intentional errors both without distraction and with right-sided distraction. Conclusion: 'Aiming' motor-preparatory brain activity may be induced by distraction in pAD subjects as compared to typical visual-motor function in controls.

Copyright $\odot 2012$ S. Karger AG, Basel
\end{abstract}

\section{Introduction}

Patients with unilateral cortical brain damage in the area of the temporoparietal cortex often exhibit an attentional disorder called spatial neglect, manifested as perceptual-attentional or motor-intentional bias toward the contralateral side of space to the brain lesion, as- 
sociated with functional deficits $[1,2]$. Neglect in patients with unilateral brain damage is readily detected by a variety of standardized measures including line bisection [1]. Patients with probable Alzheimer's disease (pAD) have bilateral cortical degeneration. On average, neural degeneration in $\mathrm{pAD}$ progresses to involve bilateral cortical temporoparietal regions, critical to maintaining spatial attentional capacity [3]. Although patients with pAD rarely manifest robust spatial attentional bias or spatial neglect, they may manifest inattention, as well as more subtle spatial deficits.

Studies of naturally occurring asymmetries in healthy persons and effects of acquired brain injuries suggest that right hemisphere systems support significant spatial bias favoring the contralateral, left body space and left visual field [2]. Both healthy and brain-injured subjects may make errors on visual-spatial tasks such as line bisection, and these errors may entail different stages of spatial processing. Subjects may be relatively unaware of one side of the line, which we could term a failure of feedback-dependent perceptual-attentional 'where' systems. Healthy young subjects may primarily manifest leftward 'where' bias when bisecting lines in near and far space [4-6]. Subjects may also make errors due to asymmetry of motor-intentional 'aiming' systems (also termed action-intentional, premotor or exploratory bias) [4, 5, 7-9]. An 'aiming' system deficit may cause subjects to fail to act in or toward one side of space, or may cause preferential action in or toward the opposite hemi-space.

Alzheimer's disease is associated with parietal cortical degeneration [10,11]. Although both parietal cortices are believed to be affected in this condition, loss of the right brain's critical support of leftward 'where' and 'aiming' orienting [12], and thus leftward bias, may decrease leftward spatial bias in subjects with this condition $[2,5,10]$.

Considering bottom-up versus top-down attentional processes provides an important theoretical framework for analyzing the nature and extent of spatial dysfunction in patients with pAD. Top-down attention can be defined as goal directed, volitional control, such as active, conscious search for a target, whereas bottom-up attention is stimulus driven and may favor novel, external stimuli with ecological relevance. A patient with a focal subcortical lesion in the left medial thalamic region reported the alarming tendency, while driving, to veer towards people or objects on the right side of the road. Barrett et al. [13] tested this patient and demonstrated that in far extrapersonal space only, she showed significant horizontal spatial bias toward a distractor. People with pAD have difficulty with both top-down and bottom-up attention, but spatial aspects of bottom-up attention in pAD may be disrupted either randomly or systematically. If bottom-up attention is disrupted systematically, as it is in post-stroke spatial neglect, this may produce functionally important spatial bias. The specific pattern of sensory perceptual inattention and motor intentional asymmetry or bias in patients with pAD may be affected by factors stimulating bottom-up attention, such as an external distractor. Spatial errors with distraction, which are present in healthy as well as impaired subjects $[4,13]$, may increase with aging and dementia [14-20]. This bottom-up bias could impair driving and other tasks that require visual-motor control. Thus, addressing spatial bias affecting attention and action might improve daily functioning in people with Alzheimer's disease, but patients cannot receive intervention if deficits are not identified.

We previously reported spatial distraction in healthy controls as a primarily 'where' perceptual-attentional phenomenon [4]. As the dorsal 'where' visual system may be more affected by neurodegenerative Alzheimer's disease than are motor association systems [21, 22], a primary 'where' spatial bias on this task may be lost in people with pAD. Alternatively, an external distractor may provoke aberrant attentional orienting in those with pAD.

The research questions for this study were:

(1) Do subjects with dementia and pAD make less leftward line bisection errors compared with age-matched controls? 

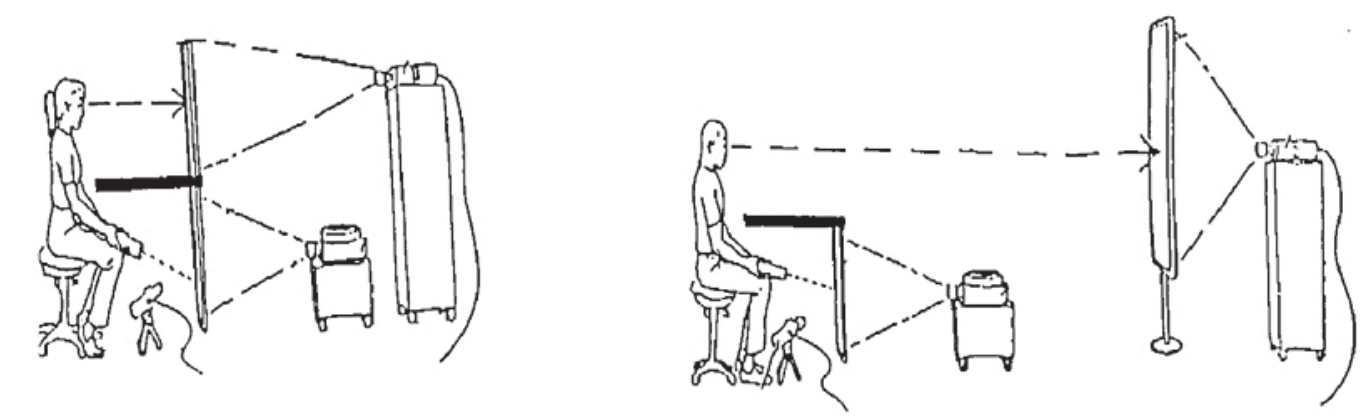

Fig. 1. Line bisection apparatus.

(2) Do subjects with dementia and pAD differ in their pattern of spatial perceptual-attentional 'where' versus motor intentional 'aiming' errors compared to controls?

(3) Do subjects with dementia and pAD demonstrate a greater distraction effect than controls while bisecting lines in the presence of a distractor?

\section{Materials and Method}

\section{Subjects}

Six subjects with dementia and pAD by NINDS ARDA criteria [23] (3 women/3 men; 1 subject with co-occurring white matter disorder classified as mixed dementia) aged 49-74 years (mean age 70.1 years) participated in this study. The Mini-Mental State Exam (MMSE) [24] was administered. Scores ranged from 24 to 30 (mean score 27.5), indicating mild pAD as the greatest severity. There were 22 aged controls ( 11 women/11 men) aged $64-83$ years (mean age 73.3 years). All subjects were right-handed by a handedness survey [25]. Control subjects had no history of neurological or psychiatric disorders, and pAD subjects had no other premorbid neurological or psychiatric diagnoses. Subjects gave their informed consent, and the study protocol was approved by the Institutional Review Board of the Pennsylvania State University, where the data was collected.

\section{Procedure}

The procedure for this study has been previously published [4]. Subjects bisected lines by directing a Laserlyte laser pointer to a self-standing, non-glare, transparent acrylic workscreen, positioned on the floor (fig. 1). The workscreen held a white sheet of paper with a black horizontal line, $22.4 \mathrm{~cm}$ long and $0.3 \mathrm{~cm}$ in width, centered on it, and was positioned $55 \mathrm{~cm}$ in front of the subjects and $15 \mathrm{~cm}$ from the floor. A white sheet draped over the subject's lap blocked a direct view of the workscreen, but a Sony (DCR-TRV730) digital 8 camera positioned below the subject's seat projected the workscreen and laser pointer position to a Sony television monitor (viewscreen). The viewscreen was located either $55 \mathrm{~cm}$ away from the subject in near space $(40 \times 30 \mathrm{~cm}$ viewscreen), or $175 \mathrm{~cm}$ away from the subject in far space $(123 \times 92.5 \mathrm{~cm}$ viewscreen $)$. In both cases, the projected line subtended a visual angle of $38.1^{\circ}$ and appeared at eye level. A videonics MXPro digital video mixer-TBC horizontally mirror-reversed the projected image in half of the trials (indirect condition), 
such that the left side of the line appeared on the right side of the monitor and vice versa. In left- and right-distractor trials, a second experimenter stood immediately to the left or right side of the monitor as the subject bisected lines. The distractor's position was randomized. The distractor stood in a neutral position and did not interact with or cue the subject in any way.

Subjects sat with their midsagittal planes aligned with the horizontal center of the workscreen and monitor, and held the laser pointer with both hands. They bisected lines in a total of 96 trials, split into 4 blocks (near-natural, near-reversed, far-natural, and far-reversed), performed in pseudorandom order. Each block was further divided into 3 distractor condition sets (left-, right-, or no-distractor), also pseudorandomized. For each trial, subjects were instructed to begin by directing the pointer to either the left or the right upper corner of the workscreen. They then moved the pointer to bisect the line, and then indicated verbally when they were satisfied with the pointer location. In subsequent trials, they alternated starting in the right or left upper corner. In the natural condition, subjects' movements corresponded to what they saw on the viewscreen, but in the reversed conditions, moving the pointer to the right resulted in apparent leftward movement on the viewscreen, and moving the pointer to the left resulted in apparent rightward movement.

\section{Measurement and Interpretation of Performance}

To score subject performance, a second camera, identical to the one above, recorded the line and the laser pointer position from the back of the workscreen, to which a metric ruler was affixed. Line bisection error from the veridical line center was measured to the nearest millimeter by an unbiased rater later viewing subjects' videotaped performance. Leftward errors were coded as negative, and rightward errors were coded as positive. Perceptual-attentional 'where' and motor-intentional 'aiming' bias were derived algebraically by comparing the natural (right-left congruent) and reversed (right-left incongruent) error conditions. We first assumed that when subjects bisected a line when feedback and performance was right-left aligned, errors reflected both 'where' and 'aiming' bias. This can be expressed algebraically as:

Errors in the natural condition $=$ where + aiming

We then assumed that when what subjects see is right-left reversed from their movements in the workspace, 'where' and perceptual attentional feedback shifts $180^{\circ}$, but feedback-forward, motor-intentional 'aiming' intentional movements do not. This can be expressed algebraically as:

Errors in the reversed condition $=$ aiming - where

This experimental logic has been previously published $[2,4,5]$. A priori, we assumed that healthy controls would make leftward 'where' spatial errors especially under conditions of left distraction [4]. In order to determine whether the current data supported this assumption, we compared 'where' bias in controls under conditions of no distraction and left-sided distraction to perfect performance, using two-tailed, one-sample t tests.

We tested the three experimental hypotheses regarding $\mathrm{pAD}$ with an analysis of variance (ANOVA), including within-subject variables: condition (natural vs. reversed), and side of distraction (left, right, or none). Group (pAD or control) was the between-subjects factor. Post hoc paired comparisons are presented in the Results section.

Hypotheses

(1) Based on our previous findings and an assumption of right-hemisphere spatial-attentional dominance, we hypothesized that controls would demonstrate primarily 'where' leftward spatial bias. 
Table 1. 'Where' error

Galletta et al.: Visual Distraction: An Altered Aiming Spatial Response in Dementia

Table 2. 'Aiming' error

\begin{tabular}{lll}
\hline Distraction type & $\begin{array}{l}\text { 'Where' error } \\
\text { pAD, mm }\end{array}$ & $\begin{array}{l}\text { 'Where' error } \\
\text { controls, mm }\end{array}$ \\
\hline Left side & -0.6 & -1.4 \\
None & -1.2 & -0.8 \\
Right side & -0.9 & -0.55 \\
\hline
\end{tabular}

\begin{tabular}{lll}
\hline Distraction type & $\begin{array}{l}\text { 'Aiming' error } \\
\text { pAD, mm }\end{array}$ & $\begin{array}{l}\text { 'Aiming' error } \\
\text { controls, mm }\end{array}$ \\
\hline Left side & 0.1 & -0.5 \\
None & 1.5 & -0.15 \\
Right side & 1.75 & -0.4 \\
\hline
\end{tabular}

(2) We predicted that 'where' spatial bias would be decreased in subjects with dementia and pAD compared to controls, given hypothesized possible loss of right-brain 'where' spatial dominance.

(3) As above, we predicted that subjects with pAD would demonstrate altered distractibility with possible loss of left-side 'where' component of distraction errors.

\section{Results}

To examine for differences in spatial bias and distractibility between subjects with dementia and healthy controls, we performed a $2 \times 2 \times 3$ ANOVA. We identified a significant three-way, group-by-bias type-by-distraction side interaction ( $\mathrm{p}=0.007$ with GreenhouseGeisser correction).

In order to further assess the interaction, we examined the effects of distraction on leftward versus rightward distraction separately. We performed two $2 \times 2 \times 2$ ANOVAs to examine differences between subjects with dementia and healthy controls in the presence of each distractor and the no-distractor conditions. Right-sided distraction showed no significant effect on either 'where' or 'aiming' bias in either group. However, left-sided distraction increased leftward bias in both subjects with dementia and controls, but 'where' and 'aiming' errors were differently affected by left distraction in the two groups. Whereas the leftward 'where' bias showed little change in the presence of a left-sided distractor (left distraction: -0.6 $\pm 2.76 \mathrm{~mm}$; no distraction: $-1.2 \pm 3.18 \mathrm{~mm}$; $=1.39$, d.f. $=5, \mathrm{p}=0.22$, n.s.), a more notable change was observed for the 'aiming' bias, which was initially rightward in the pAD group. A baseline rightward 'aiming' bias was reduced in the direction of the left distractor for the pAD group (left distraction: $0.1 \pm 1.48 \mathrm{~mm}$; no distraction: $1.5 \pm 1.74 \mathrm{~mm}$; $\mathrm{t}=-2.64$, d.f. $=5, \mathrm{p}=$ 0.05). In controls, consistent with our hypothesis, we observed a trend for left distraction to increase leftward 'where' bias (left distraction: $-1.4 \pm 2.38 \mathrm{~mm}$; no distraction: $-0.8 \pm 2.49$ $\mathrm{mm} ; \mathrm{t}=-1.74$, d.f. $=21, \mathrm{p}=0.096)$. See tables 1 and 2 and figures 2 and 3 for illustration.

We previously observed asymmetric distraction in healthy subjects and stroke survivors $[4,6]$. Thus, to examine whether asymmetric distraction occurred in one group, but not in the other, and whether our a priori hypothesis of loss of left-sided distraction in subjects with dementia was supported, we performed post hoc pairwise comparisons. We examined distraction effects for the control subjects and subjects with dementia by comparing perfor- 
Fig. 2. Line bisection 'where' bias with and without distraction.

Fig. 3. Line bisection 'aiming' bias with and without distraction.
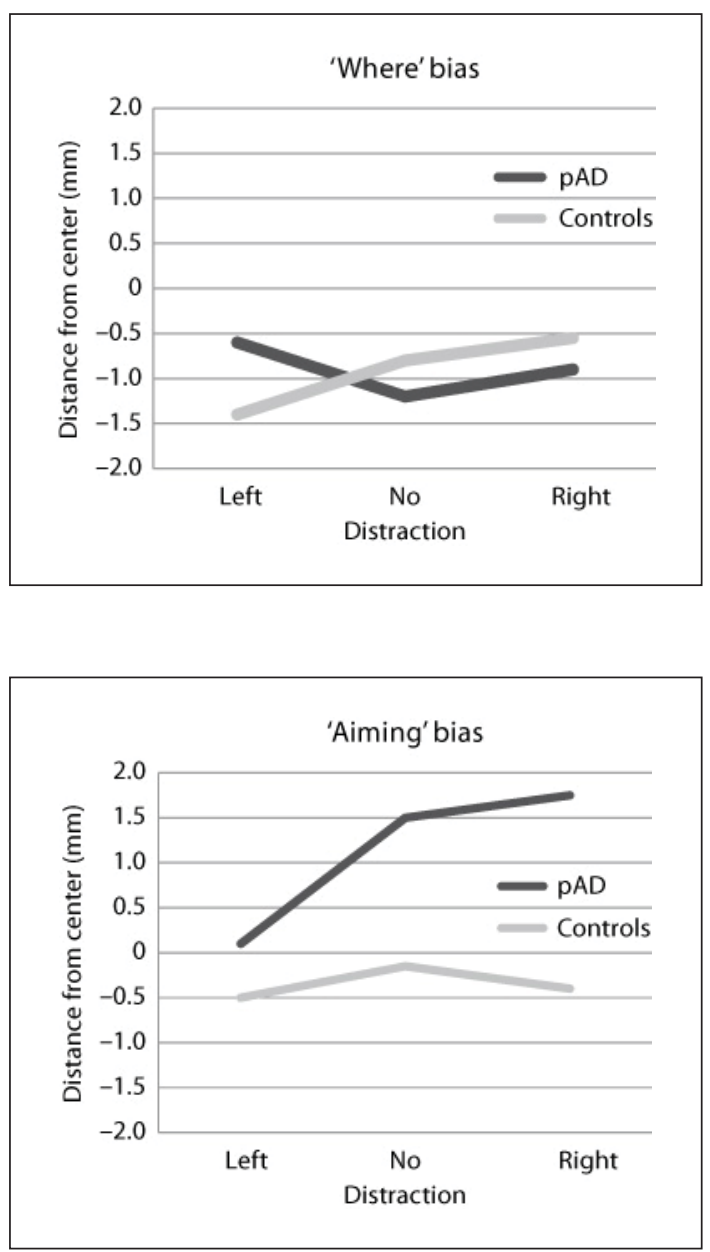

mance without distraction to performance with left- and right-distraction using two-tailed, paired-sample t tests. We performed separate 'where' and 'aiming' bias comparisons, since in our previous studies it appeared that distraction primarily affects 'where' errors [4]. We were particularly interested in whether a left-distractor effect, increasing leftward 'where' errors as compared with testing without distraction, would be present in controls but not in subjects with dementia.

Thus, it is possible that the three-way interaction between group, bias type, and distraction reflected a primary effect of left-sided distraction on 'where' bias in controls, whereas left-sided distraction primarily affected 'aiming' bias in subjects with dementia. Right-sided distraction exerted no significant effect on either 'where' or 'aiming' bias in controls or participants with dementia (all comparisons: $\mathrm{p}>0.24$, n.s.).

Main effects of distraction $(\mathrm{p}=0.15)$ and bias $(\mathrm{p}=0.07)$ did not reach significance (table 1; fig. 2).

\section{Discussion}

Our a priori hypotheses were partly supported, but with important differences bearing further investigation. Although healthy controls did demonstrate leftward errors with left distraction, we only observed a trend toward leftward 'where' distraction when we compared 
left- and no-distraction conditions, which did not reach significance after correction for multiple comparisons. The small sample size could have also affected this. However, a highly significant three-way interaction between group, distraction side, and 'where'/'aiming' bias type suggested that distraction affects different types of bias in subjects with dementia as compared with normal controls.

In a surprising finding, we did not note that subjects with dementia had increased errors in the presence of a novel distractor. When subjects with $\mathrm{pAD}$ were asked to bisect lines, they were not distracted by the presence of a person standing near the stimulus. This is similar to the effect reported by Drago et al. [26], who implemented two types of distraction, a bottomup meaningless condition and a top-down condition requiring the participant to name the color of the lateral distractor. Their meaningless, bottom-up condition, similar to our distractor condition, consisted of a light placed lateral to the line without specific response relevant to the participant. They observed that in the top-down condition, in which participants were required to name the color of the light, $\mathrm{pAD}$ participants made increased spatial errors in the direction of the stimulus (manifested distraction). This was attributed to difficulty with executive re-allocation of spatial attention.

However, our results, unlike those of Drago et al. [26], support a difference in motor preparatory spatial system processing in pAD in response to distraction. In our experiment, subjects with pAD manifested rightward 'aiming' bias at baseline, which moved leftward under conditions of leftward distraction. Thus, although left-sided distraction in our pAD subjects was not associated with any increase in 'where' bias, it was associated with leftward increases in 'aiming' bias, a phenomenon not observed in controls.

In a normal individual, the predominant response to left-sided distraction may be reallocation of perceptual-attentional 'where' spatial resources toward the left-sided spatial region in which novel stimuli appear. Motor-intentional resources relevant to 'aiming' output systems may be unaffected in the normal brain, until top-down gating releases a response. We do not know why leftward distraction may affect 'aiming' spatial systems in pAD.

It is not clear why left-sided distraction might induce changes in 'aiming' bias in subjects with dementia and pAD, rather than inducing changes in 'where' bias; however, this may be consistent with exaggerated response to top-down spatial cueing observed by Drago et al. [26]. It is also possible that subcortical activity induced by distracting stimuli via structures such as the superior colliculus may interact differently with spatial systems in dementia and pAD than it does in normal visual-motor function. Neurodegeneration affecting the superior colliculus in $\mathrm{pAD}[10]$ may reduce perceptual-attentional 'where' responsivity to distraction. However, the deep superior colliculus, mediating rapid action decisions on novel environmental stimuli or unexpected events, relevant in 'aiming' bias, may be less affected [27]. Thus, the deep, motor superior colliculus may still be responsive to input from premotor frontal lobe 'aiming' responses activated by distracting stimuli or by top-down spatial response sets [28]. In pAD, this may result in a response to distraction which may be manifested more by an 'aiming' motor intentional abnormality than by a 'where' perceptual-attentional allocation asymmetry. Such an asymmetry of collicular activation could be small in magnitude and not normally measurable except in sensitive testing situations such as the video line bisection task.

If an 'aiming' spatial asymmetry in pAD in response to leftward distraction reflects a small, measurable spatial environmental dependency $[13,29,30]$, it could be a source of errors during complex spatial activities such as driving. It also might appear early in the course of pAD; thus, it is important to gain a better understanding of the mechanism of this phenomenon and how any impact on safety might be prevented, reduced, or eliminated. Drago et al.'s [26] study suggests that leftward 'aiming' distraction in pAD might be increased by top-down motor cuing in the direction of a distractor, and reduced by top-down motor cu- 
ing in the opposite direction. If the deep, motor colliculus plays an important role in these errors, they might also be more marked in a body-centered than head-centered reference frame [27, 31]. Further experiments on these topics are indicated.

\section{Acknowledgements}

Work on this study was partially supported by the Kessler Foundation (E.E.G., A.M.B., and A.H.L.) and the National Institutes of Health (E.E.G., A.M.B.: R01NS05580, K24HD062647).

\section{References}

1 Heilman KM, Valenstein E: Mechanisms underlying hemispatial neglect. Ann Neurol 1979;5:166170.

-2 Barrett AM, Burkholder S: Monocular patching in subjects with right-hemisphere stroke affects perceptual-attentional bias. J Rehabil Res Dev 2006;43:337-346.

-3 Giovagnoli AR, Erbetta A, Reati F, Bugiani O: Differential neuropsychological patterns of frontal variant frontotemporal dementia and Alzheimer's disease in a study of diagnostic concordance. Neuropsychologia 2008;46:1495-1504.

-4 Garza JP, Eslinger P, Barrett AM: Perceptual-attentional and motor-intentional bias in near and far space. Brain Cogn 2008;68:9-14.

-5 Schwartz RL, Adair JC, Na D, Williamson DJG, Heilman KM: Spatial bias: attentional and intentional influence in normal subjects. Neurology 1997;48:234-242.

-6 Jewell G, McCourt ME: Pseudoneglect: a review and meta-analysis of performance factors in line bisection tasks. Neuropsychologia 2000;38:93-110.

-7 Bisiach E, Geminiani G, Berti A, Rusconi ML: Perceptual and premotor factors of unilateral neglect. Neurology 1990;40:1278-1281.

-8 Coslett HB, Bowers D, Fitzpatrick E, Haws B, Heilman KM: Directional hypokinesia and hemispatial inattention in neglect. Brain 1990;113:475-486.

-9 Mapstone M, Steffenella TM, Duffy JD: A visuospatial variant of mild cognitive impairment: getting lost between aging and AD. Neurology 2003;60:802-808.

-10 Boxer AL, Rankin KP, Miller BL, Schuff N, Weiner M, Gorno-Tempini ML, Rosen HJ: Cinguloparietal atrophy distinguishes Alzheimer disease from semantic dementia. Arch Neurol 2003;60:949956.

-11 Scahill RI, Schott JM, Stevens JM, Rossor MN, Fox NC: Mapping the evolution of regional atrophy in Alzheimer's disease: unbiased analysis of fluid-registered serial MRI. Proc Natl Acad Sci USA 2002;99:4703-4707.

-12 Heilman KM, Van Den Abell T: Right hemisphere dominance for attention: the mechanism underlying hemispheric asymmetries of inattention (neglect). Neurology 1980;30:327-330.

-13 Barrett AM, Schwartz RL, Crucian GP, Manho K, Heilman KM: Attentional grasp in far extrapersonal space after thalamic infarction. Neuropsychologia 2000;38:778-784.

$\checkmark 14$ Mutter SA, Goedert KM: Frequency discrimination vs frequency estimation: adult age differences and the effect of divided attention. J Gerontol B Psychol Sci Soc Sci 1997;52:P319-P328.

15 Chao LL, Knight RT: Prefrontal deficits in attention and inhibitory control with aging. Cereb Cortex 1997;7:63-69.

-16 Madden DJ, Pierce TW, Allen PA: Adult age differences in the use of distractor homogeneity during visual search. Psychol Aging 1996;11:454-474.

-17 Carlson MC, Hasher L, Zacks RT, Connelly SL: Aging, distraction, and the benefits of predictable location. Psychol Aging 1995;10:427-436.

18 Hasher L, Zacks RT: Working memory, comprehension and aging: a review and a new view; in Bower GH (ed): The Psychology of Learning and Motivation: Advances in Research and Theory. San Diego, Academic Press, 1988, pp 193-225. 
-19 Schneider NG, Gritz ER, Jarvick ME: Age differences in simple paced tasks of attention and perception. Gerontology 1979;23:142-147.

-20 Layton B: Perceptual noise and aging. Psychol Bull 1975;82:875-883.

-21 Benson DF, Davis J, Snyder BD: Posterior cortical atrophy. Arch Neurol 1988;45:789-793.

-22 Pietrini P, Furey ML, Graff-Radford N, Freo U, Alexander GE, Grady CL, et al: Preferential metabolic involvement of visual cortical areas in a subtype of Alzheimer's disease: clinical implications. Am J Psychiatry 1996;153:1261.

-23 McKhann G, Drachman D, Folstein M, et al: Clinical diagnosis of Alzheimer's disease: report of NINCDS-ADRDA Work Group under the auspices of the Department of Health and Human Services Task Force on Alzheimer's Disease. Neurology 1984;34:939-944.

-24 Folstein MF, Folstein SE, McHugh PR: 'Mini-mental state'. A practical method for grading the cognitive state of patients for the clinician. J Psychiatr Res 1975;12:189-198.

-25 Raczkowski D, Kalat JW, Nebes R: Reliability and validity of some handedness questionnaire items. Neuropsychologia 1974;12:43-47.

-26 Drago V, Foster PS, Ferri R, Arico D, Lanuzza B, Heilman KM: Distractability and Alzheimer disease: the neglected phenomenon. J Alzheimers Dis 2008;15:1-10.

-27 Iseki E, Matsushita M, Kosaka K, Kondo H, Ishii T, Amano N: Distribution and morphology of brain stem plaques in Alzheimer's disease. Acta Neuropathol 1989;78:131-136.

-28 Lunenburger L, Kleiser R, Stuphorn V, Miller LE, Hoffmann KP: A possible role of the superior colliculus in eye-hand coordination. Prog Brain Res 2001;134:109-125.

-29 Werner W, Hoffmann KP, Dannenberg S: Anatomical distribution of arm-movement-related neurons in the primate superior colliculus and underlying reticular formation in comparison with visual and saccadic cells. Exp Brain Res 1997;115:206-216.

- 30 Kwon SE, Heilman K: Ipsilateral neglect in a patient following a unilateral frontal lesion. Neurology 1991;41:2001-2004.

-31 Lhermitte F: Human autonomy of the frontal lobes. Part II: patient behaviour in complex and social situations: the 'environmental dependency syndrome'. Ann Neurol 1986;19:335-343.

-32 Neggers SFW, Bekkering H: Gaze anchoring to a pointing target is present during the entire pointing movement and is driven by a non-visual signal. J Neurophysiol 2001;86:961-970. 